\title{
Can superhorizon cosmological perturbations explain the acceleration of the universe?
}

\author{
Christopher M. Hirata ${ }^{1, *}$ and Uroš Seljak ${ }^{1,2}$ \\ ${ }^{1}$ Department of Physics, Jadwin Hall, Princeton University, Princeton, New Jersey 08544, USA \\ ${ }^{2}$ International Center for Theoretical Physics, Strada Costiera 11, 34014 Trieste, Italy
}

(Received 29 March 2005; published 3 October 2005)

\begin{abstract}
We investigate the recent suggestions by Barausse et al. and Kolb et al. that the acceleration of the universe could be explained by large superhorizon fluctuations generated by inflation. We show that no acceleration can be produced by this mechanism. We begin by showing how the application of Raychaudhuri equation to inhomogeneous cosmologies results in several "no go" theorems for accelerated expansion. Next we derive an exact solution for a specific case of initial perturbations, for which application of the Kolb et al. expressions leads to an acceleration, while the exact solution reveals that no acceleration is present. We show that the discrepancy can be traced to higher-order terms that were dropped in the Kolb et al. analysis. We proceed with the analysis of initial value formulation of general relativity to argue that causality severely limits what observable effects can be derived from superhorizon perturbations. By constructing a Riemann normal coordinate system on initial slice we show that no infrared divergence terms arise in this coordinate system. Thus any divergences found previously can be eliminated by a local rescaling of coordinates and are unobservable. We perform an explicit analysis of the variance of the deceleration parameter for the case of single-field inflation using usual coordinates and show that the infrared-divergent terms found by Barausse et al. and Kolb et al. cancel against several additional terms not considered in their analysis. Finally, we argue that introducing isocurvature perturbations does not alter our conclusion that the accelerating expansion of the universe cannot be explained by superhorizon modes.
\end{abstract}

DOI: 10.1103/PhysRevD.72.083501

PACS numbers: $98.80 . \mathrm{Jk}, 98.80 . \mathrm{Cq}$

\section{INTRODUCTION}

There are now several lines of evidence pointing toward an accelerating expansion of the universe. These include the luminosity distance-redshift relation measured from Type Ia supernovae (SN Ia) [1-6]; the combination of the angular diameter distance to the surface of last scattering and the physical matter density $\Omega_{m} H_{0}^{2}$ measured from the cosmic microwave background (CMB) with the low values of $\Omega_{m} H_{0}$ favored by large-scale structure data [711]; and, most recently, the integrated Sachs-Wolfe effect [12-19]. It is well known that such an accelerating expansion is impossible if one makes the following three assumptions:

(1) The strong energy condition (SEC) holds, i.e. the density and isotropic part of the pressure seen by all observers on timelike trajectories satisfy $\rho+$ $3 p \geq 0$

(2) the universe is described by general relativity (GR); and

(3) the universe is homogeneous and isotropic, in particular, the Friedmann-Robertson-Walker (FRW) metric is applicable.

Any explanation for the acceleration of the universe must drop at least one of these three assumptions. Usually either assumption (1) or (2) is dropped. In such a case, we use the term "dark energy" to describe any SEC-violating matter field, and "modified gravity" to denote any departure from

*Electronic address: chirata@princeton.edu
GR. These explanations for the acceleration could be considered unsatisfying since there is presently no other experimental motivation for modifications to GR, and the matter fields normally considered in cosmology, including baryonic matter, photons, neutrinos, and cold dark matter (CDM) all obey the SEC. In either case, new physics must be invoked. In contrast, it is observed that assumption (3) is not exactly valid in the real universe. Therefore several recent papers $[20,21]$ have asked whether in fact the "backreaction" from these perturbations to the universe can explain the acceleration, without dropping the SEC or GR.

The purpose of this paper is to examine the recent suggestions by Barausse et al. [20] (hereafter BMR) and Kolb et al. [21] (hereafter KMNR) that perturbations on scales larger than the Hubble length can explain the acceleration. In particular, these papers suggest that the time evolution of these large-scale perturbations produce a large variance of the deceleration parameter $q$. Since potential perturbations at the horizon scale are of order $\sim 10^{-5}$, one would expect the fluctuations in $q$ to be of this order, however KMNR argues that corrections due to very large-scale modes (hundreds of $e$-folds outside the horizon) can cause the standard deviation of $q$ to be $\gg 10^{-5}$. In particular, for spectral index $n_{s} \leq 1$ they claim that the corrections from very large-scale modes contain an infrared divergence. If the variance is very large, this could mimic dark energy and cause an apparent acceleration. Indeed, the existence of perturbations on scales well beyond the horizon is likely in the context of inflation 
[22-24], since the inflaton field may have been in the "slow roll" regime for many more $e$-folds than the minimum of $\sim 60$ necessary to solve the horizon problem. This paper focuses on the possibility of accelerated expansion from superhorizon perturbations; we do not consider mechanisms based on subhorizon physics here.

We argue that in fact no acceleration is produced. Our arguments can be summarized as follows:

(i) The result that GR + SEC forbids accelerating expansion is more general than the FRW cosmology. The application of the Raychaudhuri equation to inhomogeneous cosmologies immediately yields several "no go" theorems for accelerating expansion with zero vorticity, depending on how acceleration is defined. One of these theorems rules out the KMNR mechanism. We treat the definition of the deceleration parameter $q$ and the conditions under which $q<0$ is possible in Sec. II.

(ii) In Sec. III, we examine the physical reason why KMNR found that their deceleration parameter becomes negative, in contradiction to our conclusion in Sec. II B. In particular, we examine one configuration of initial perturbations that can be solved exactly; we show that in this case, the discrepancy arises because of higher-order terms in the perturbative expansion of $\Psi$ [their Eq. (7)] that were dropped in their analysis. Once the full result for $\Psi$ is used, the deceleration parameter $q$ never becomes negative. The results of Sec. III are less general than those of Sec. II, since they only apply to a specific configuration. Nevertheless they provide important insight into the calculation of KMNR.

(iii) Causality severely limits what observable effects can be derived from superhorizon perturbations. If one constructs an initial 3-dimensional hypersurface $\Sigma$ at the end of inflation, then any observable quantities can in principle be determined purely from the values of the initial perturbations on $\Sigma \cap$ $J^{-}(\mathcal{O})$, where $J^{-}(\mathcal{O})$ is the causal past of the observer. There may be perturbations on very large scales, e.g. due to the early stages of inflation, but one can only observe these to the extent that they affect the initial conditions on $\Sigma \cap J^{-}(\mathcal{O})$. We show in the first half of Sec. IV that the observable effect of superhorizon perturbations found by BMR - including the infrared divergence - is in fact due to a local rescaling of the coordinates caused by the particular choice of gauge for the superhorizon modes.

(iv) In Sec. IV C, we calculate the contribution of very long-wavelength modes to the deceleration parameter in single-field inflation models. We find that this contribution vanishes when all effects, including non-Gaussianity and loop corrections to the scalar power spectrum, are taken into account.
This means that the long-wavelength modes in single-field inflationary models cannot produce a large variance of the deceleration parameter.

Section II shows that regardless of the potential perturbations, "acceleration" as defined by KMNR cannot be obtained without sacrificing the SEC or GR or invoking vorticity (which is not present in KMNR and is not predicted by inflation), while Sec. IV argues that there is no infrared divergence and that the fluctuations in $q$ at the horizon scale are indeed of order $\sim 10^{-5}$. Sections III and IVC deal with the more specific issues of why KMNR found acceleration and infrared divergence, respectively, and address technical points about their calculation. Note that our arguments involving the "no go" theorems are logically independent from the arguments involving causality. Either one rules out the KMNR mechanism as the explanation for the accelerating expansion of the universe.

The results of Sec. IVC are specific to single-field inflation, and do not apply to other models. For example, in multifield inflation large isocurvature perturbations are possible, which can produce large variances in the deceleration parameter when one averages over different portions of the universe [25]. However the analysis of Sec. II still applies to these cases, i.e. for several of the definitions of the deceleration parameter there is no possibility of obtaining $q<0$ without rejecting the SEC or GR.

The notation used in this paper is as follows: the metric signature will be -+++ , with $h_{i j}$ denoting the spatial metric. Greek indices will range over $\{0,1,2,3\}$ whereas Latin indices range only over the spatial directions $\{1,2,3\}$. In cases where we do explicit calculations with the metric, we will use the synchronous CDM-comoving gauge, in which $g_{00}=-1, g_{0 i}=0$, and the CDM particles move along curves of constant spatial coordinates $x^{k}$ (we show in Sec. II that this gauge exists in any scenario, including KMNR, where the CDM vorticity vanishes). Symmetrization of indices will be defined by $A_{(a b)}=\frac{1}{2}\left(A_{a b}+\right.$ $A_{b a}$ ), and similarly for antisymmetrization, which is denoted with square brackets $A_{[a b]}$. In the model of KMNR, there are only scalar perturbations present initially, and the spatial metric becomes

$$
h_{i j}\left(t, x^{k}\right) \rightarrow a^{2}(t) e^{-10 \varphi\left(x^{k}\right) / 3} \delta_{i j}
$$

at early times when the perturbation mode of interest is outside the horizon (but after the end of inflation); we use this as the definition of $\varphi$. The overdot will denote derivatives with respect to the physical time coordinate $t$; we will not do any calculations with the conformal time in this paper. The analyses of Secs. III and IV are done on a flat FRW background with a scale factor $a(t) \propto t^{2 / 3}$ normalized to $a\left(t_{0}\right)=1$ today. 


\section{DECELERATION PARAMETER}

In order to make a statement about the universe accelerating, it is necessary to have a precise definition of the deceleration parameter that makes sense in non-FRW cosmologies. In the recent backreaction papers, several definitions have been proposed. We consider these here.

\section{A. Definitions}

The simplest definition is based on the congruence of timelike geodesics formed by the CDM particles with 4velocity $u^{\mu}$; one may define a local Hubble constant

$$
H_{1}=\frac{1}{3} \nabla_{\mu} u^{\mu}
$$

and then a deceleration parameter

$$
q_{1}=-1-H_{1}^{-2} u^{\mu} \nabla_{\mu} H_{1} .
$$

Here $u^{\mu} \nabla_{\mu}$ is the Lagrangian proper time derivative $d / d t_{\text {proper }}$ associated with a CDM particle. Also Eq. (2) implies that $H_{1}$ is related to the expansion tensor $\theta_{\mu \nu}$ via $\theta \equiv \theta_{\mu}^{\mu}=3 H_{1}$. Since the CDM particles follow geodesics, the expansion tensor is purely spatial in the CDM particle's frame, i.e. it is orthogonal to $\mathbf{u}: \theta_{\mu \nu} u^{\nu}=\theta_{\mu \nu} u^{\mu}=0$ (c.f. Eq. 9.2.2 of [26]). We will need to decompose $\theta_{\mu \nu}$ into the usual irreducible tensors

$$
\theta_{\mu \nu}=\frac{1}{3} \theta\left(g_{\mu \nu}+u_{\mu} u_{\nu}\right)+\omega_{\mu \nu}+\sigma_{\mu \nu},
$$

where $g_{\mu \nu}+u_{\mu} u_{\nu}$ is the spatial metric (where "spatial" is defined by an observer sitting on a CDM particle), $\omega_{\mu \nu}=$ $\nabla_{[\mu} u_{\nu]}$ is a $3 \times 3$ purely spatial antisymmetric tensor with 3 independent components, and $\sigma_{\mu \nu}$ is a tracelesssymmetric spatial tensor with 5 independent components.

In the special case where the vorticity 2-form $\omega=\frac{1}{2} d \mathbf{u}$ is zero, we can integrate the exact 1 -form $\mathbf{u}$ and write $\mathbf{u}=$ $-d t$ for some locally defined 0 -form $t$. (In most cases of interest, the spacetime is simply connected and $t$ is defined globally.) The congruence of CDM particles is then said to be irrotational. If one labels the CDM particles with coordinates $x^{i}$, then $\mathbf{u}=-d t$ implies that the covariant components of $\mathbf{u}$ are $u_{\mu}=(-1,0,0,0)$. However since the particle spatial coordinates are constant, $u^{i}=0$; normalization $u_{\mu} u^{\mu}=-1$ requires $u^{0}=1$ and $u^{\mu}=(1,0,0,0)$. The requirement that these components of $u_{\mu}$ and $u^{\mu}$ be consistent forces $g_{00}=-1$ and $g_{0 i}=0$, and the line element of the metric then becomes

$$
d s^{2}=-d t^{2}+h_{i j}\left(x^{k}, t\right) d x^{i} d x^{j} .
$$

A straightforward computation shows that

$$
H_{1}=\frac{1}{3} \Gamma_{\mu \nu}^{\mu} u^{\nu}=\frac{1}{6} h^{i j} \partial_{t} h_{i j}=\frac{1}{6} \partial_{t} \operatorname{lndeth},
$$

and this is recognized as the definition of the variable
" $H\left(t, x^{i}\right)$ " used by Ref. [25]. It is also equivalent to the operational definition used by KMNR since our deth is equivalent to their $\bar{a}^{6} e^{-6 \Psi_{s}}$ and they ignore the small-scale perturbation modes $\Psi_{s}$. In the latter case where a deceleration parameter is used, it is defined as

$$
-\frac{\bar{a} \partial_{t}^{2} \bar{a}}{\left(\partial_{t} \bar{a}\right)^{2}}=-1-\frac{\partial_{t} H_{1}}{H_{1}^{2}}=q_{1},
$$

i.e. the deceleration parameter $\bar{q}$ used by KMNR is equivalent to our $q_{1}$.

The Hubble and deceleration parameters $H_{1}$ and $q_{1}$ as defined above are functions of both the time coordinate $t$ and the spatial coordinates $x^{i}$. It is sometimes proposed to measure spatially averaged values of the Hubble constant,

$$
H_{2}(t)=\frac{\int_{\mathcal{V}} H_{1} \sqrt{h} d^{3} x}{\int_{\mathcal{V}} \sqrt{h} d^{3} x}=\frac{1}{3} \partial_{t} \ln \int_{\mathcal{V}} \sqrt{h} d^{3} x
$$

and

$$
q_{2}(t)=\frac{\int_{\mathcal{V}} q_{1} \sqrt{h} d^{3} x}{\int_{\mathcal{V}} \sqrt{h} d^{3} x}
$$

Note that, unlike the case of $q_{1}$, we have $q_{2} \neq$ $-1-\dot{H}_{2} / H_{2}^{2}$.

The deceleration parameters $q_{1}$ and $q_{2}$ are convenient because they are easy to compute in many cosmological models, avoiding the complicated process of tracing null geodesics and estimating luminosity distances used by BMR. The latter, of course, is their principal shortcoming: $q_{1}$ and $q_{2}$ do not correspond to the observables in any of today's major cosmological probes. The famous Hubble plot of $z$ versus $d_{L}$ allows one to measure $H_{1}$, however extragalactic astronomy is only $\sim 10^{2}$ years old and this is a woefully inadequate baseline with which to directly measure the time derivative $\dot{H}_{1}$ and hence the deceleration parameter $q_{1}$. We also cannot cover the entire universe with astronomers so as to measure spatially averaged quantities such as $\mathrm{H}_{2}$ or $q_{2}$.

Since SN Ia luminosity distances are one of the major pieces of evidence for the accelerating expansion of the universe, one way to make contact with observations is to calculate the luminosity distance-redshift relation. This was first done in the context of backreaction by BMR, who expanded the luminosity distance $d_{L}$ as a Taylor series in redshift,

$$
d_{L}=H_{3^{\prime}}^{-1} z+\frac{1-q_{3^{\prime}}}{2 H_{3^{\prime}}} z^{2}+O\left(z^{3}\right) .
$$

As defined here, the Hubble and deceleration parameters $H_{3^{\prime}}$ and $q_{3^{\prime}}$ depend not only on the observer's spacetime coordinates $t, x^{i}$, but also on the direction of observation (RA and Dec), because in a perturbed universe there is no reason why the $d_{L}-z$ relation should be the same in every direction. BMR then went on to average these over solid angle to obtain 


$$
H_{3}=\left\langle H_{3^{\prime}}\right\rangle_{4 \pi} \text { and } q_{3}=\left\langle q_{3^{\prime}}\right\rangle_{4 \pi} .
$$

A related procedure is to write Eq. (10) as a function $z\left(d_{L}\right)$,

$$
z=H_{3^{\prime}} d_{L}-\frac{1}{2} H_{3^{\prime}}^{2}\left(1-q_{3^{\prime}}\right) d_{L}^{2}+O\left(d_{L}^{3}\right) .
$$

This motivates the definition

$$
\langle z\rangle_{4 \pi}=H_{4} d_{L}-\frac{H_{4}^{2}\left(1-q_{4}\right)}{2} d_{L}^{2}+O\left(d_{L}^{3}\right),
$$

where we take the angular average over the redshift at fixed $d_{L}$ rather than averaging the Taylor coefficients. This procedure has the advantage of making sense in slightly more general conditions. In particular, if $\theta_{i j}$ has a direction $n^{i}$, not necessarily an eigenvalue, where $\theta_{i j} n^{i} n^{j}=0$, then $H_{3^{\prime}}=0$ in that direction and $q_{3^{\prime}}$ in Eq. (10) is undefined. Then $q_{3}$ is not defined either. In contrast, Eq. (13) breaks down only under the more restricted condition that $H_{4}=$ 0 , i.e. that the angular average of $H_{3^{\prime}}$ vanishes. As we will see in the next section, it is also easier to prove theorems about $q_{4}$ because one can avoid angular averages with quantities such as $H_{3^{\prime}}$ in the denominator.

One can relate $H_{4}$ and $q_{4}$ to $H_{3}$ and $q_{3}$ by considering the angular average of the coefficients of $d_{L}$ and $d_{L}^{2}$ in Eq. (12),

$$
\begin{aligned}
H_{4} & =\left\langle H_{3^{\prime}}\right\rangle_{4 \pi}=H_{3} \quad \text { and } \\
H_{4}^{2}\left(1-q_{4}\right) & =\left\langle H_{3^{\prime}}^{2}\left(1-q_{3^{\prime}}\right)\right\rangle_{4 \pi} .
\end{aligned}
$$

Thus we always have $H_{3}=H_{4}$. If $H_{3^{\prime}}$ is isotropic, then it cancels out in the second line implying $q_{3}=q_{4}$, but $q_{3}$ and $q_{4}$ need not be identical in general.

\section{B. Conditions for acceleration}

Having written down several definitions of the deceleration parameter, one can ask: under what conditions can the universe be accelerating, i.e. under what conditions is $q<0$ possible?

The condition for acceleration is easiest to derive in the case of $q_{1}$. We begin with the Raychaudhuri equation,

$$
\frac{d \theta}{d t}=-\frac{\theta^{2}}{3}-\sigma_{\mu \nu} \sigma^{\mu \nu}+\omega_{\mu \nu} \omega^{\mu \nu}-R_{\mu \nu} u^{\mu} u^{\nu},
$$

where $R_{\mu \nu}$ is the Ricci tensor. Using this Eq. (3) can be rewritten as

$$
H_{1}^{2} q_{1}=\frac{1}{3}\left(\sigma_{\nu}^{\mu} \sigma_{\mu}^{\nu}-\omega_{\nu}^{\mu} \omega_{\mu}^{\nu}\right)+\frac{1}{3} R_{\mu \nu} u^{\mu} u^{\nu} .
$$

The Einstein equation allows us to rewrite the last term in terms of the matter content in the CDM particles' rest frame,

$$
H_{1}^{2} q_{1}=\frac{1}{3}\left(\sigma_{\nu}^{\mu} \sigma_{\mu}^{\nu}-\omega_{\nu}^{\mu} \omega_{\mu}^{\nu}\right)+\frac{4 \pi G}{3}(\rho+3 p),
$$

where $\rho=T^{00}$ is the total density and $p=\frac{1}{3} T_{i}^{i}$ is the isotropic pressure. Since $\sigma$ is purely spatial in the local Lorentz frame of a CDM particle, its square is semipositive definite, $\sigma_{\nu}^{\mu} \sigma_{\mu}^{\nu} \geq 0$. Thus we immediately see that if the strong energy condition (SEC) is satisfied (so that $\rho+$ $3 p \geq 0)$ and there is no vorticity $(\omega=0)$, it follows that $q_{1} \geq 0$.

The vorticity $\omega$ plays a key role in all of the "no go" theorems regarding accelerated expansion, as well as in the existence of the synchronous CDM-comoving gauge [Eq. (5)] and the spatially averaged deceleration $q_{2}$, so it is worth exploring when it can be nonzero. It can be shown (see e.g. Eq. 9.2.14 of [26]) that if $\omega=0$ at one time then it remains zero at all future times (at least until orbit crossings appear). In particular, the existence of the synchronous CDM-comoving gauge implies $\mathbf{u}=-d t$ and $\omega=d \mathbf{u}=-d^{2} t=0$, so if the synchronous CDMcomoving gauge exists at early times then $\omega=0$. KMNR explicitly use the synchronous CDM-comoving gauge in their calculations, so their model has zero vorticity. This means that the "no go" theorems that $q_{1} \geq 0$ applies to KMNR; since they use $q_{1}$ as their definition for the deceleration parameter, the KMNR mechanism cannot produce acceleration. A corollary is that $q_{1} \rightarrow-1$ in KMNR must be an artifact of the perturbative expansion. We will return to this point in Sec. III.

Since $q_{2}$ is a spatial average of $q_{1}$, it follows trivially that in the absence of vorticity or SEC violation, $q_{2} \geq 0$.

The deceleration parameters $q_{3}$ and $q_{4}$ in terms of luminosity distances can be related to observations. The simplest way to do this is to consider the photon trajectories that arrive at the observer $\mathcal{O}$, and find the luminosity distance $d_{L}(v)$ and redshift $z(v)$ as a function of affine parameter $v$. The photon is assumed to arrive at the observer at affine parameter $v=0$ with unit energy, i.e. its 4momentum $\mathbf{k}$ satisfies $k_{\mu} u^{\mu}=-1$ at $v=0$. We will assume that the vorticity of the CDM particles vanishes so that we can use the synchronous CDM-comoving gauge [Eq. (5)]. In this gauge, the " 0 " direction coincides with the CDM particle 4-velocity, so the purely spatial nature of the expansion tensor $\theta_{\mu \nu}$ can be used: $\theta_{00}=\theta_{0 i}=\theta_{i 0}=$ 0 , and we will henceforth simply write $\theta_{i j}$. We also define the photon's 3-velocity at the observer $n_{i}=k_{i}(v=0)$, and note that this is a unit vector since the photon is massless. Note that $v<0$ on the observer's past light cone.

The redshift associated with a particular point on the photon's trajectory is simply $1+z(v)=k^{0}(v)$, since $k^{0}(v)$ is the photon's energy as measured by the CDM particle and the observer sees energy 1 . We can expand $k^{0}(v)$ to second order using the geodesic equation,

$$
\frac{d x^{\alpha}}{d v}=k^{\alpha} \quad \text { and } \quad \frac{d k^{\alpha}}{d v}=-\Gamma_{\mu \nu}^{\alpha} k^{\mu} k^{\nu} .
$$

The derivatives of $k^{0}$ are then 


$$
\frac{d k^{0}}{d v}=-\Gamma_{i j}^{0} k^{i} k^{j}=-\frac{1}{2} \dot{h}_{i j} k^{i} k^{j}
$$

since $\Gamma_{00}^{0}=\Gamma_{0 i}^{0}=0$ in the synchronous gauge, and

$$
\frac{d^{2} k^{0}}{d v^{2}}=-\frac{1}{2} k^{\alpha}\left(\partial_{\alpha} \dot{h}_{i j}\right) k^{i} k^{j}+\dot{h}_{i j} k^{i} \Gamma_{\mu \nu}^{j} k^{\mu} k^{\nu} .
$$

Plugging in the Christoffel symbols yields

$$
\begin{aligned}
k^{0}= & 1+\frac{d k^{0}}{d v} v+\frac{d^{2} k^{0}}{d v^{2}} \frac{v^{2}}{2} \\
= & 1-\frac{1}{2} \dot{h}_{i j} n^{i} n^{j} v+\left[-\frac{n^{i} n^{j}}{2}\left(\ddot{h}_{i j}+n^{k} \dot{h}_{i j, k}\right)+\frac{\dot{h}_{i j} n^{j}}{2}\right. \\
& \left.\times\left(2 h^{i k} \dot{h}_{k l} n^{l}-h^{i l} h_{m k, l} n^{k} n^{m}+2 h^{i l} h_{m l, k} n^{k} n^{m}\right)\right] \frac{v^{2}}{2} .
\end{aligned}
$$

Introducing the extrinsic curvature $K_{i j}=-\frac{1}{2} \dot{h}_{i j}$, and its 3dimensional covariant derivative $K_{i j \mid k}$,

$$
K_{(i j \mid k)}=K_{(i j, k)}+K_{(i}^{l} h_{j k), l}-2 h_{l(j, k} K_{i)}^{l},
$$

yields the redshift

$$
z=K_{i j} n^{i} n^{j} v+\left(\dot{K}_{i j}+K_{i j \mid k} n^{k}+4 K_{i}^{k} K_{k j}\right) n^{i} n^{j} \frac{v^{2}}{2} .
$$

Since $\mathbf{u}$ is the unit normal to the hypersurfaces of constant $t$, it follows that $K_{i j}=-\theta_{i j}$ (c.f. Eq. 9.3 .19 of [26]).

The luminosity distance to a spherical source whose surface is at affine parameter $v_{s}$ is given by Eq. (2.14) of Ref. [27]

$$
d_{L}\left(v_{s}\right)=R \frac{A\left(v_{s}\right)}{A(0)}\left[1+z\left(v_{s}\right)\right],
$$

where $A$ is the photon amplitude (normalized by $T^{\mu \nu}=$ $A^{2} k^{\mu} k^{\nu}$ ) and $R$ is the radius of the source; the amplitude $A$ is given by

$$
A \propto \exp \left(-\frac{1}{2} \int \hat{\theta} d v\right)
$$

where $\hat{\theta}=\nabla_{\mu} k^{\mu}$ is the photon expansion. The photon expansion near the source can be expanded as

$$
\hat{\theta}=\frac{2}{v-\left(v_{s}+\Delta v_{s}\right)}+O\left[v-\left(v_{s}+\Delta v_{s}\right)\right],
$$

where $-\Delta v_{s}$ is the difference affine parameter from the source's center to its surface at radius $R$; this can be shown by the same argument leading to BMR's Eq. (A27). This affine parameter can be found by noting that $k^{\mu}=$ $d x^{\mu} / d v$. Here the photon's energy at the source is $k^{0}=$ $1+z$ and the time it takes to travel from the center to the surface is $R$, so

$$
\Delta v_{s}=-\frac{k^{0}}{\Delta t}=-\frac{1+z}{R} .
$$

Also, integration of Eq. (25) using Eq. (26) gives

$$
\frac{A\left(v_{s}\right)}{A(0)}=\frac{-\Delta v_{s}}{-v_{s}-\Delta v_{s}}=\frac{1+z}{-v_{s} R},
$$

where the last equality uses $\left|\Delta v_{s}\right| \ll\left|v_{s}\right|$. This yields

$$
d_{L}\left(v_{s}\right)=-v_{s}\left[1+z\left(v_{s}\right)\right]^{2}+O\left(v_{s}^{3}\right) .
$$

Comparing to Eq. (23) gives the result, to second order, that

$$
z=-\frac{K_{i j} n^{i} n^{j} d_{L}}{(1+z)^{2}}+\left(\dot{K}_{i j}+K_{i j \mid k} n^{k}+4 K_{i}^{k} K_{k j}\right) n^{i} n^{j} \frac{d_{L}^{2}}{2},
$$

or

$$
\begin{aligned}
z= & -K_{i j} n^{i} n^{j} d_{L}-2\left(K_{i j} n^{i} n^{j}\right)^{2} d_{L}^{2} \\
& +\left(\dot{K}_{i j}+K_{i j \mid k} n^{k}+4 K_{i}^{k} K_{k j}\right) n^{i} n^{j} \frac{d_{L}^{2}}{2} .
\end{aligned}
$$

We can then calculate the deceleration parameter $q_{4}$ by comparing Eq. (31) to Eq. (13). The $K_{i j \mid k}$ term drops out in the angular averaging, and we have $\left\langle n^{i} n_{i}\right\rangle_{4 \pi}=\frac{1}{3}$, so we get

$$
H_{4}=-\frac{1}{3} K
$$

where $K=K_{i}^{i}$. Note that this implies $H_{4}=H_{1}$. The deceleration parameter is

$$
q_{4}=1+H_{4}^{-2}\left[-4 K_{i j} K_{k l} \frac{h^{(i j} h^{k l)}}{5}+\frac{1}{3} \dot{K}_{i j} h^{i j}+\frac{4}{3} K_{j}^{i} K_{i}^{j}\right] .
$$

This can be simplified by separating $K$ into its isotropic and anisotropic pieces, $K_{j}^{i}=-H_{4} \delta_{j}^{i}-\sigma_{j}^{i}$. Then we find

$$
q_{4}=1+\frac{\dot{K}_{i j} h^{i j}}{3 H_{4}^{2}}+\frac{4}{5} H_{4}^{-2} \sigma_{j}^{i} \sigma_{i}^{j}
$$

A further simplification comes from the formula for $\dot{K}_{i j}$ (Eq. 21.82 of Ref. [28]),

$$
R^{0}{ }_{i 0 j}=-\left(\dot{K}_{i j}+K_{i k} K_{j}^{k}\right)
$$

the antisymmetry property of the Riemann tensor coupled with the Einstein equation allows us to write

$$
h^{i j} R^{0}{ }_{i 0 j}=R_{0}^{0}=8 \pi G\left(T_{0}^{0}-\frac{1}{2} T_{\alpha}^{\alpha}\right)=-4 \pi G(\rho+3 p),
$$

hence

$$
\dot{K}_{i j} h^{i j}=4 \pi G(\rho+3 p)-3 H_{4}^{2}-\sigma_{j}^{i} \sigma_{i}^{j} .
$$

Substitution into Eq. (34) yields

$$
q_{4}=\frac{4 \pi G(\rho+3 p)}{3 H_{4}^{2}}+\frac{7 \sigma_{j}^{i} \sigma_{i}^{j}}{15 H_{4}^{2}} .
$$


Once again, we see that if the SEC holds then $q_{4} \geq 0$. Thus by this definition too a universe with only matter cannot accelerate.

The deceleration parameter $q_{3}$ is only well defined for a positive-definite spatial expansion tensor $\theta_{i j}$. It is also more difficult to work with than $q_{4}$ : from Eq. (12), we find

$$
q_{3^{\prime}}=1+2 \frac{\left.\left(\partial^{2} z / \partial d_{L}^{2}\right)\right|_{d_{L}=0}}{\left(\partial z /\left.\partial d_{L}\right|_{d_{L}=0}\right)^{2}}
$$

The presence of variables in the denominator of this equation makes it very difficult to prove theorems about the angular average $q_{3}=\left\langle q_{3^{\prime}}\right\rangle_{4 \pi}$. We do know from Eq. (14) that $H_{4}=H_{3}$. Since the mean of the square is always at least the square of the mean, this implies

$$
H_{4}^{2} \leq\left\langle H_{3^{\prime}}^{2}\right\rangle_{4 \pi} \text {. }
$$

Combining Eq. (40) with the second line of Eq. (14) yields

$$
\left\langle H_{3^{\prime}}^{2} q_{3^{\prime}}\right\rangle_{4 \pi}=\left\langle H_{3^{\prime}}^{2}\right\rangle_{4 \pi}-H_{4}^{2}+H_{4}^{2} q_{4} \geq H_{4}^{2} q_{4} .
$$

If the SEC holds then $q_{4} \geq 0$ and it follows that $\left\langle H_{3^{\prime}}^{2} q_{3^{\prime}}\right\rangle_{4 \pi} \geq 0$. This inequality does allow for the possibility that $q_{3}=\left\langle q_{3^{\prime}}\right\rangle_{4 \pi}<0$. This can happen if there is an anisotropic expansion tensor (i.e. $\sigma \neq 0$ ) so that $\left\langle H_{3^{\prime}}^{2} q_{3^{\prime}}\right\rangle_{4 \pi} \neq H_{3}^{2}\left\langle q_{3^{\prime}}\right\rangle_{4 \pi}$ and the universe is "accelerating" with $q_{3^{\prime}}<0$ in some directions $n^{i}$. Nevertheless even for an anisotropic expansion, the inequality $\left\langle H_{3^{\prime}}^{2} q_{3^{\prime}}\right\rangle_{4 \pi} \geq 0$ implies that if there are accelerating directions $\left(q_{3^{\prime}}<0\right)$ then there must be decelerating directions $\left(q_{3^{\prime}}>0\right)$ as well.

\section{ACCELERATION OF UNDERDENSE REGIONS?}

KMNR concluded that an underdense region of a postinflationary universe filled with only dark matter can appear to accelerate (according to $q_{1}$ ) due to the influence of superhorizon perturbations. However, with CDM only the SEC should hold, and after inflation there should be no vorticity. In Sec. II B, we showed that the SEC and zero vorticity imply $q_{1} \geq 0$. These conclusions are obviously inconsistent. The purpose of this section is to show, in a particular case that can be solved exactly, that the reason for the inconsistency is that KMNR dropped higher-order perturbative terms in their gravitational potential $\Psi_{\ell}$. These terms, when incorporated into their Eq. (10), result in a deceleration $q_{1}$ that never becomes negative.

The particular case that we shall consider here is the potential

$$
\varphi=\frac{3}{5} \ln \left(1-\frac{1}{4} C|x|^{2}\right),
$$

where $|x|^{2}=\left(x^{1}\right)^{2}+\left(x^{2}\right)^{2}+\left(x^{3}\right)^{2}$ and $C$ is a constant. In order for the conclusions derived below to be valid, it is sufficient by causality for $\varphi$ to take on the above value inside our horizon. Also, by Birkhoff's theorem, if the potential is spherically symmetric and takes on the form of Eq. (42) for $|x|$ less than some radius $R$, the future evolution at $|x|<R$ is as computed below.

Since for $C>0$ we have $\nabla^{2} \varphi<0$, the potential Eq. (42) can be used as a model for an underdense region. Not all underdense regions look like Eq. (42), however this potential can be solved and it is conceptually useful for understanding why the higher-derivative terms dropped in KMNR are in fact important. The spherically symmetric underdensity and overdensity have been studied extensively as models of voids in large-scale structure [29] and the formation and growth of collapsed objects [30] respectively, however these studies were not aimed at understanding backreaction. Our aim here is to understand the implications of the spherical underdensity for the KMNR mechanism.

Substitution of the potential in Eq. (42) into Eq. (1) yields initial conditions for the spatial metric,

$$
h_{i j}\left(t, x^{k}\right)=\frac{a^{2}(t)}{\left(1-\frac{1}{4} C|x|^{2}\right)^{2}}\left[\delta_{i j}+O\left(t^{2 / 3}\right)\right] .
$$

This is simply the metric for a 3-dimensional open universe with spatial curvature $-C$ and a coordinate system given by the stereographic projection, analytically continued to the case of negative curvature. The future evolution of this universe is equivalent to an open FRW universe and thus can thus immediately be written down:

$$
d s^{2}=-d t^{2}+\frac{a_{\text {open }}^{2}(t)}{\left(1-\frac{1}{4} C|x|^{2}\right)^{2}} d x^{i} d x^{i},
$$

where $a_{\text {open }}(t)$ satisfies the Friedmann equation for an open universe, which has the parametric solution

$$
\left\{\begin{array}{l}
a_{\text {open }}=\Xi \sqrt{C}(\cosh \eta-1), \\
t=\Xi(\sinh \eta-\eta)
\end{array}\right.
$$

where $\Xi$ is a constant and $\eta$ is the parameter. Comparison of Eqs. (43) and (44) shows that at early times, $a_{\text {open }}(t) / a(t) \rightarrow 1$. Since the evolution of $a(t)$ is simply that of the Einstein-de Sitter universe,

$$
a(t)=\left(\frac{3}{2} H_{0} t\right)^{2 / 3},
$$

we can use this as the early-time limit of $a_{\text {open }}(t)$ to determine $\Xi$,

$$
\Xi=\frac{1}{2} H_{0}^{2} C^{-3 / 2} \text {. }
$$

KMNR defined the metric perturbation variable $\Psi$ according to

$$
d s^{2}=-d t^{2}+a^{2}(t) e^{-2 \Psi\left(x^{i}, t\right)} d x^{i} d x^{i} .
$$

Comparison of Eq. (44) with Eq. (48) then allows us to determine the full nonperturbative evolution of $\Psi$ for the potential $\varphi$ of Eq. (42): 


$$
\Psi\left(x^{i}, t\right)=\ln \left(1-\frac{1}{4} C|x|^{2}\right)-\ln \frac{a_{\text {open }}(t)}{a(t)} .
$$

The first term in this result is independent of time and, as correctly argued by KMNR, it does not affect $q_{1}$. The second term can be evaluated by expressing $\eta$ in terms of $t$ and hence $a(t)$ in the second line of Eq. (45) and then finding $a_{\text {open }}$ using the first line. The functional form $\eta(t)$ cannot be expressed analytically, but a power series can be developed via reversion of series,

$$
\eta=\left(\frac{6 t}{\Xi}\right)^{1 / 3}-\frac{1}{60}\left(\frac{6 t}{\Xi}\right)+\frac{1}{1400}\left(\frac{6 t}{\Xi}\right)^{5 / 3}+\ldots
$$

Substitution into the Taylor expansion of $a_{\text {open }}(t)=$ $\Xi \sqrt{C}(\cosh \eta-1)$ gives

$$
\begin{aligned}
a_{\mathrm{open}}(t)= & a(t)+\frac{C}{5 H_{0}^{2}} a^{2}(t)-\frac{3 C^{2}}{175 H_{0}^{4}} a^{3}(t) \\
& +O\left(C^{3} H_{0}^{-6} a^{4}\right) .
\end{aligned}
$$

We are interested in the expression for $a_{\text {open }}$ as a perturbation series in $C$, since $\varphi \rightarrow 0$ when $C \rightarrow 0$. Equation (51) provides this series, although it turns out to also be a power series in $a$. [The reason for this is that $C$ has units of length ${ }^{-2}$, and $H_{0}$ is the only other quantity containing units of length once we have eliminated $\rho_{0}$ using the Friedmann equation. Thus $C$ and $H_{0}$ appear together in the combination $C / H_{0}^{2}$ when expanding $a_{\text {open. }}$. Furthermore, there is an invariance associated with the choice of epoch of the observer: if one places the observer at a later time $\xi t$ instead of $t$, then the scale factors $a$ and $a_{\text {open }}$ are multiplied by $\xi^{-2 / 3}$ (since $a \propto t^{2 / 3}$ ), $H_{0}$ is multiplied by $\xi^{-1}$, and the change in definition of comoving length multiplies the "curvature" constant $C$ by $\xi^{-4 / 3}$, hence $C / H_{0}^{2}$ is multiplied by $\xi^{2 / 3}$. The invariance with respect to $\xi$ forces the terms in the above expansion to take the form $\left(C / H_{0}^{2}\right)^{j} a^{j+1}$. Thus the expansion in $C$ turns out to also be an expansion in $a$.] Substitution into Eq. (49) gives

$$
\begin{aligned}
\Psi\left(x^{i}, t\right)= & \ln \left(1-\frac{1}{4} C|x|^{2}\right)-\frac{C}{5 H_{0}^{2}} a(t)+\frac{13 C^{2}}{350 H_{0}^{4}} a^{2}(t) \\
& +O\left(C^{3} H_{0}^{-6} a^{3}\right) .
\end{aligned}
$$

Aside from the irrelevant time-independent term, $\Psi\left(x^{i}, t\right)$ depends only on the time. Technically the perturbation series in Eq. (52) has a finite radius of convergence of $\left|\mathrm{CH}_{0}^{-2} a\right|<\left(\frac{3}{2} \pi\right)^{2 / 3}$ (see the appendix), but if one had the full perturbative expansion one could analytically continue it to later times.

Our result for the evolution of the gravitational potential should be compared to Eq. (7) of KMNR, which after plugging in Eq. (42) yields

$$
\Psi\left(x^{i}, t\right)=\ln \left(1-\frac{1}{4} C|x|^{2}\right)-\frac{C}{5 H_{0}^{2}} a(t)+\frac{C^{2}|x|^{2}}{60 H_{0}^{2}} a(t) .
$$

The constant in this equation agrees with Eq. (52). The first-order perturbation terms (i.e. $\propto C$ ) also agree. Beyond first order in $C$, however, two discrepancies appear. One is the spurious term in Eq. (53) proportional to $C^{2}$ that has spatial dependence. We will consider $\Psi$ and $q$ the spatial origin $x^{i}=0$, where the spurious term vanishes. [This is also where the KMNR calculation is most likely to be valid, since they neglected terms containing $(\nabla \varphi)^{2}$, and $\nabla \varphi=0$ at the origin for our specific potential, Eq. (42).] The other discrepancy is that Eq. (53) is missing the higher-order spatially independent terms proportional to $(C a)^{2},(C a)^{3}$, etc. The $(C a)^{n}$ term has $2 n$ derivatives in it and presumably are not included in Eq. (7) of KMNR because they dropped "higher-derivative" terms.

We finally consider the implications of the difference between Eqs. (49) and (53) for the determination of the deceleration parameter $q_{1}$. This is given by Eq. (6) of KMNR,

$$
q_{1}=-1+\frac{\frac{3}{2}+H^{-2} \ddot{\Psi}}{\left(1-H^{-1} \dot{\Psi}\right)^{2}} .
$$

Here we have dropped the subscript on $\Psi_{\ell}$ since $\Psi=\Psi_{\ell}$ at the level of approximation in KMNR, and $H$ represents the unperturbed Hubble constant. According to Eq. (53), the value of $q_{1}$ at the spatial origin $x^{i}=0$ is

$$
q_{1}(\mathrm{KMNR})=-1+\frac{\frac{3}{2}+\frac{1}{10} H_{0}^{-2} C a}{\left(1+\frac{1}{5} H_{0}^{-2} C a\right)^{2}} .
$$

According to the full nonperturbative calculation, and defining $H_{\text {open }}(t)=\dot{a}_{\text {open }}(t) / a_{\text {open }}(t)$, we find

$$
\dot{\Psi}=H(t)-H_{\text {open }}(t) \quad \text { and } \quad \ddot{\Psi}=\dot{H}(t)-\dot{H}_{\text {open }}(t) .
$$

Substituting into Eq. (54) yields

$$
q_{1}(\text { true })=-1-\frac{\dot{H}_{\text {open }}(t)}{H_{\text {open }}^{2}(t)},
$$

where $H(t)$ completely drops out if one uses the identity $\dot{H}=-\frac{3}{2} H^{2}$. We can use Eq. (45) to find $H_{\text {open }}(t)$, which gives

$$
H_{\text {open }}(t)=A^{-1} \frac{\sinh \eta}{\cosh \eta-1}
$$

and

$$
q_{1}(\text { true })=\frac{1}{1+\cosh \eta} .
$$

Equations (55) and (59) have some features in common: they both approach $1 / 2$ at early times, and as the universe 
expands they decrease, as expected for an underdense region of the universe. However the long-time behavior is different: as $t \rightarrow \infty, q_{1}(\mathrm{KMNR}) \rightarrow-1$, as noted by KMNR, whereas $\eta \rightarrow \infty$ and hence $q_{1}$ (true) $\rightarrow 0$. We thus see explicitly that no acceleration is possible in this case and that the result found by KMNR is a consequence of neglecting higher-order terms in their analysis: if the full perturbation series had been calculated (and analytically continued to late times), KMNR would have found no acceleration.

\section{SUPERHORIZON ADIABATIC PERTURBATIONS}

BMR have recently analyzed the effect of superhorizon adiabatic perturbations on the $d_{L}-z$ relation. In particular they considered the value of the isotropically averaged deceleration parameter $q_{3}\left(\left\langle q_{0}\right\rangle_{\Omega}\right.$ in their notation). They found a large contribution to the variance of $q_{3}$ coming from the interaction of infrared and ultraviolet modes. In this section we rederive this result by selecting an appropriate new coordinate system. This method illuminates the physical origin of the large $\operatorname{Var} q_{3}$ found by BMR, namely, that BMR assumed the ultraviolet modes to be statistically homogeneous, isotropic, and Gaussian in their coordinate system with the cosmic power spectrum $P_{\varphi}(k)$. Because of the infrared perturbations, this coordinate system differs from the locally FRW coordinate system erected by an observer, and the power spectrum of ultraviolet modes seen by a specific observer differs from $P_{\varphi}(k)$. In Sec. IV C, we consider the $\varphi$ field generated by single-field inflation, and show that in this case the BMR infrared divergences are canceled by non-Gaussian features and loop corrections to the power spectrum.

The KMNR mechanism makes use of this infrared divergence. Specifically, they argue that in some models of inflation, there is a very large variance of the deceleration parameter $q_{1}$ resulting from the interaction of infrared and horizon-scale modes. Within the context of single-field inflation, or indeed any mechanism in which small-scale perturbations are laid down after the large-scale perturbations have exited the horizon and frozen out, there is then no infrared divergence. We discuss the implications for KMNR in Sec. IV D.

\section{A. Gauge transformation; causality}

The initial state of any system in classical general relativity is described by a spatial metric $h_{i j}$ on the initial hypersurface, its time derivative, i.e. extrinsic curvature $K_{i j}$, and the appropriate variables $X_{a}$ to describe the matter fields. These quantities are not all independent since they must satisfy the energy and momentum constraints. It is also well-known that two distinct sets of initial conditions $\left\{h_{i j}, K_{i j}, X_{a}\right\}$ may actually correspond to the same spacetime because they differ by a gauge transformation, which may involve either (i) a reparametrization of the initial hypersurface, or (ii) selection of a different spacelike hypersurface as the initial slice. We will focus our analysis on using gauge transformations of type (i) since (ii) is more difficult to analyze.

If one considers an initial hypersurface $\Sigma$ after the end of inflation, one need only specify the initial data on the portion of $\Sigma$ that can be seen by the observer, $\mathcal{O}$, i.e. one needs initial data on $\Sigma \cap J^{-}(\mathcal{O})$. In the unperturbed standard CDM model with the usual initial hypersurface (a surface of constant cosmic time), $\Sigma \cap J^{-}(\mathcal{O})$ is a closed ball with comoving radius less than the horizon size $\chi_{h}$,

$$
\chi_{h}=\int_{0}^{1} \frac{d a}{a^{2} H(a)}=2 H_{0}^{-1},
$$

where we have used $H(a) \propto a^{-3 / 2}$. In a non-FRW universe the nature of $\Sigma \cap J^{-}(\mathcal{O})$ is more complicated. Nevertheless, Cauchy stability [31] implies that, for sufficiently small perturbations, the observables seen by $\mathcal{O}$ can be uniquely determined from the initial data on any open set $\mathcal{U}$ in $\Sigma$ with $\mathcal{U} \supseteq \Sigma \cap J^{-}(\mathcal{O}$, FRW $)$, where $J^{-}(\mathcal{O}, \mathrm{FRW})$ is the causal past of the observer assuming the FRW metric (see Fig. 1). In particular this means that given such a $\mathcal{U}$, one can calculate the observables seen by $\mathcal{O}$ to any order in perturbation theory. It is also easy to construct $\mathcal{U}$ : any open ball centered at the origin and extending out to coordinate radius $R_{\mathcal{U}}>\chi_{h}$ will do. We assume $R_{U}=2 \chi_{h}$ for definiteness.

The initial hypersurface considered by BMR has a spatial metric

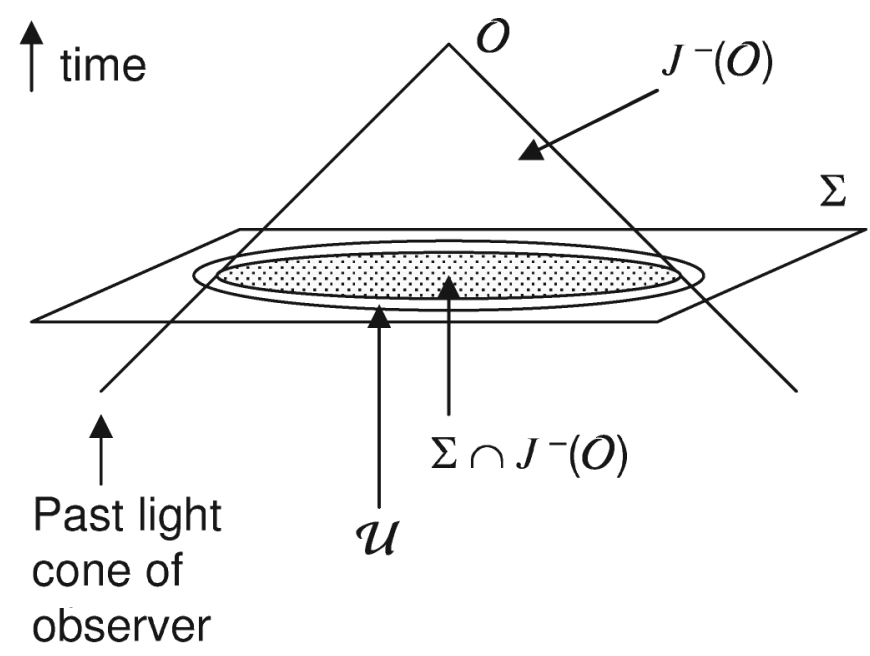

FIG. 1. The regions of spacetime described in the text. Initial data are supplied on the Cauchy hypersurface $\Sigma$. Causality implies that the observables seen by observer $\mathcal{O}$ can depend only on the initial data within the shaded region $\Sigma \cap J^{-}(\mathcal{O})$. Within the context of perturbation theory around an FRW spacetime, the perturbations to the observables can be determined given initial data within any open set $\mathcal{U}$ containing $\Sigma \cap$ $J^{-}(\mathcal{O}$, FRW $)$. Superhorizon perturbations can only be observed to the extent that they affect the initial data inside $\mathcal{U}$. 


$$
\begin{aligned}
h_{i j}\left(t_{\Sigma}, x^{k}\right) & =a^{2}\left(t_{\Sigma}\right) \gamma_{i j}\left(t_{\Sigma}, x^{k}\right) \\
& =a^{2}\left(t_{\Sigma}\right) e^{-10 \varphi\left(x^{k}\right) / 3}\left[\delta_{i j}+O\left(t^{2 / 3}\right)\right]
\end{aligned}
$$

and extrinsic curvature

$$
\begin{aligned}
K_{i j}\left(t_{\Sigma}, x^{k}\right) & =-\frac{1}{2} \dot{h}_{i j}\left(t_{\Sigma}, x^{k}\right) \\
& =-H\left(t_{\Sigma}\right) h_{i j}\left(t_{\Sigma}, x^{k}\right)+O\left(K_{i j} t^{2 / 3}\right),
\end{aligned}
$$

where we write $O\left(K_{i j} t^{2 / 3}\right)$ to mean that the fractional correction is of order $t^{2 / 3}$. Instead of $x^{i}$ coordinates we want to construct a coordinate system which is explicitly locally inertial. We will therefore impose a gauge transformation to new coordinates $y^{i}\left(x^{j}\right)$ such that at early times, $h_{i j}$ is very close to $a^{2} \delta_{i j}$ (without the conformal factor). One realization of such a coordinate system is to construct geodesics in all directions from the center and decompose their tangent vectors in terms of a chosen basis: the resulting coordinates are the Riemann normal coordinates.

We begin by splitting $\varphi$ in Eq. (61) into a large-scale and a small-scale piece,

$$
\varphi\left(x^{i}\right)=\varphi_{L}\left(x^{i}\right)+\varphi_{S}\left(x^{i}\right) .
$$

We will work to first order in $\varphi_{S}$ and in the wave number $k_{L}$ of the large-scale modes. That is, any terms containing $\varphi_{S}^{2}$ or $k_{L}^{2} \varphi_{L} \sim \nabla \nabla \varphi_{L}$ will be dropped. Note that the split into "long" and "short" wavelengths here differs from the split $\Psi=\Psi_{\ell}+\Psi_{s}$ in KMNR: we are including both, and are doing the separation so that $k_{L}$ and $\varphi_{S}$ are the expansion parameters, whereas in KMNR $\Psi_{s}$ is dropped.

Next we construct a Riemann normal coordinate system on the initial slice, centered at $x^{i}=0$, with respect to the large-scale metric $\gamma_{L}$. The construction of such a coordinate system begins with the choice of an orthonormal basis of three vectors $\left\{\left(\mathbf{e}_{\bar{a}}\right)^{i}\right\}_{\bar{a}=1}^{3}$ tangent to $\Sigma$ at the point $\left(t_{\Sigma}, 0,0,0\right)$. (We use the barred indices $\bar{a}$ to denote the components associated with these basis vectors and with the $\mathbf{y}$ coordinate system that will be defined based on them.) Such a basis is not unique; here we choose

$$
e_{\bar{a}}^{i}=\left[\gamma_{L}^{-1 / 2}(\mathbf{0})\right]_{\bar{a}}^{i},
$$

where one takes the $-1 / 2$ power of the $3 \times 3$ matrix of covariant components of $\gamma_{L i j}$. The Riemann normal coordinates are then constructed via

$$
x^{i}\left(y^{\bar{j}}\right)=\exp _{0}\left\{e_{\bar{j}}^{i} y^{\bar{j}}\right\},
$$

where exp represents the exponential geodesic mapping. In our case, the argument of the exponential simplifies to the vector

$$
e_{\bar{j}}^{i} y^{\bar{j}}=\left[\gamma_{L}^{-1 / 2}(\mathbf{0})\right]_{\bar{j}}^{i} y^{\bar{j}}=e^{-5 \varphi_{L}(\mathbf{0}) / 3} y^{i} .
$$

The geodesic equation of the $\gamma_{L}$ metric can be found from its Christoffel symbols,

$$
\begin{aligned}
\frac{d^{2} x^{i}}{d \lambda^{2}} & =-\Gamma_{j k}^{i} \frac{d x^{j}}{d \lambda} \frac{d x^{k}}{d \lambda} \\
& =\frac{5}{3} e^{10 \varphi_{L} / 3}\left(-\frac{\partial \varphi_{L}}{\partial x^{i}}\left|\frac{d x^{j}}{d \lambda}\right|^{2}+2 \frac{\partial \varphi_{L}}{\partial x^{j}} \frac{d x^{i}}{d \lambda} \frac{d x^{j}}{d \lambda}\right) .
\end{aligned}
$$

Solving this equation to second order in $y^{i}$ subject to the initial conditions $\mathbf{x}(\lambda=0)=\mathbf{0}$ and $\mathbf{x}^{\prime}(\lambda=0)$ given by Eq. (66) gives

$$
\begin{aligned}
x^{i}= & x^{i}(\lambda=1) \\
= & e^{10 \varphi_{L}(\mathbf{0}) / 3} y^{i}+\frac{5}{6} e^{20 \varphi_{L}(\mathbf{0}) / 3}\left[-|y|^{2} \varphi_{L, i}(\mathbf{0})\right. \\
& \left.+2 y^{i} y^{j} \varphi_{L, j}(\mathbf{0})\right] .
\end{aligned}
$$

The metric $\gamma_{L}$ in Riemann normal coordinates defined with an orthonormal basis is $\delta_{\bar{i} \bar{j}}$ at 0 and its first spatial derivatives vanish (see e.g. Sec. 11.6 of [28]). Thus

$$
\gamma_{L \bar{i} \bar{j}}\left(y^{\bar{k}}\right)=\delta_{\bar{i} \bar{j}}+O\left(k_{L}^{2} \varphi_{L}|y|^{2}\right),
$$

where $k_{L}^{2} \varphi_{L}$ is the second spatial derivative of $\varphi_{L}$, which is suppressed for long-wavelength perturbations. Note that within the set $\mathcal{U},|y|^{2}$ is at most of order $H_{0}^{-1}$. The full metric $\gamma_{\bar{i} \bar{j}}=e^{10 \varphi_{S} / 3} \gamma_{L \bar{i} \bar{j}}$ is then

$$
\gamma_{\bar{i} \bar{j}}\left(y^{\bar{k}}\right)=e^{10 \varphi_{S}[\mathbf{x}(\mathbf{y})] / 3} \delta_{\bar{i} \bar{j}}+O\left(k_{L}^{2} \varphi_{L}|y|^{2}\right) .
$$

We may now calculate the deceleration parameter in the new coordinate system. BMR has found the deceleration parameter to be, to first order in $\varphi$,

$$
q_{3}=\frac{1}{2}+\frac{5}{9} H_{0}^{-2} \nabla_{\mathbf{x}}^{2} \varphi+O\left(\varphi^{2}\right)
$$

Since Eq. (70) is now simply the Euclidean metric with a first-order perturbation, we can apply Eq. (71) in the $\mathbf{y}$ coordinate system to yield

$$
q_{3}=\frac{1}{2}+\frac{5}{9} H_{0}^{-2} \nabla_{\mathbf{y}}^{2} \varphi_{S}+O\left(\varphi_{S}^{2}, H_{0}^{-2} k_{L}^{2} \varphi_{L}\right) .
$$

The order of the error is now $H_{0}^{-2} k_{L}^{2} \varphi_{L}$ since Eq. (70) had error $O\left(k_{L}^{2} \varphi_{L}|y|^{2}\right)$ and causality requires that we can only observe out to distances $|y|<R u \sim O\left(H_{0}^{-1}\right)$. Thus in Riemann normal coordinate system, which is constructed locally and does not make reference to anything farther from the observer than the horizon, the result for the deceleration parameter is extremely simple and large amplitude long-wavelength modes have no impact on the deceleration parameter.

\section{B. Deceleration parameter in $x$ coordinates}

BMR expressed the deceleration parameter $q_{3}$ in the $\mathbf{x}$ coordinate system rather than $\mathbf{y}$. We have already shown that the analysis in $\mathbf{y}$ coordinates results in Eq. (72) and that superhorizon modes cannot affect the deceleration parameter. Nevertheless, we wish to compare our result to BMR, 
who did the calculation in the $\mathbf{x}$ coordinates. Since the final result for any observable cannot depend on the choice of coordinates we must obtain the same answer as suggested by Eq. (72). We will show below that this is indeed the case and is a result of a precise cancellation between several terms, some of which were not considered by BMR or KMNR.

We can recover all terms in the deceleration parameter up to order $k_{L} \varphi_{L}$ by using Eq. (68) to turn $\nabla_{\mathbf{y}}^{2} \varphi_{S}$ into derivatives with respect to $\mathbf{x}$ :

$$
\nabla_{\mathbf{y}}^{2} \varphi_{S}=-\frac{5}{3} e^{20 \varphi_{L} / 3} \varphi_{L, i} \varphi_{S, i}+e^{10 \varphi_{L} / 3} \nabla_{\mathbf{x}}^{2} \varphi_{S}
$$

Plugging into Eq. (72) yields

$$
\begin{aligned}
q_{3} & =\frac{1}{2}+\frac{5}{9} H_{0}^{-2}\left(-\frac{5}{3} e^{20 \varphi_{L} / 3} \varphi_{L, i} \varphi_{S, i}+e^{10 \varphi_{L} / 3} \nabla_{\mathbf{x}}^{2} \varphi_{S}\right) \\
& =\frac{1}{2}+\frac{2}{H_{0}^{2}}\left(\frac{5}{18} \nabla_{\mathbf{x}}^{2} \varphi_{S}-\frac{25}{54} \varphi_{L, i} \varphi_{S, i}+\frac{25}{27} \varphi_{L} \nabla_{\mathbf{x}}^{2} \varphi_{S}\right),
\end{aligned}
$$

where in the last line we have worked to order $\varphi_{L}$. This should be compared to Eq. (36) of BMR, which reads

$$
\begin{aligned}
q_{3}= & \frac{1}{2}+\frac{2}{H_{0}^{2}}\left(\frac{5}{18} \nabla_{\mathbf{x}}^{2} \varphi-\frac{25}{54}\left|\nabla_{\mathbf{x}} \varphi\right|^{2}+\frac{25}{27} \varphi \nabla_{\mathbf{x}}^{2} \varphi\right) \\
& +O\left(H_{0}^{-4}\right) .
\end{aligned}
$$

In particular, the infrared-divergent term $\varphi \nabla_{\mathbf{x}}^{2} \varphi$ identified by BMR appears in Eq. (74). The $\varphi_{L, i} \varphi_{S, i}$ term is not infrared divergent (unless $n_{s} \leq-1$ ), nevertheless it is first order in $k_{L}$ so it appears here; the coefficient is double that in BMR because of the factor of 2 in the binomial expansion of $(\nabla \varphi)^{2}$. The variance of the divergent term is

$$
\begin{aligned}
\operatorname{Var}\left(\varphi \nabla_{\mathbf{x}}^{2} \varphi\right)= & {\left[\int P_{\varphi}(k) \frac{d^{3} \mathbf{k}}{(2 \pi)^{3}}\right]\left[\int k^{4} P_{\varphi}(k) \frac{d^{3} \mathbf{k}}{(2 \pi)^{3}}\right] } \\
& +\int k_{3}^{2} k_{4}^{2} T_{\varphi}\left(\left\{\mathbf{k}_{j}\right\}\right) \delta\left(\sum_{j=1}^{4} \mathbf{k}_{j}\right) \prod_{j=1}^{4} \frac{d^{3} \mathbf{k}_{j}}{(2 \pi)^{3}},
\end{aligned}
$$

where $T_{\varphi}$ is the connected trispectrum of the potential in the $\mathbf{x}$ coordinate system. BMR argues that since inflation produces Gaussian initial conditions, the trispectrum term drops out, and if $n_{s} \leq 1$, where $P_{\varphi}(k) \propto k^{n_{s}-4}$, there is an infrared divergence. We show in the next section that this divergence disappears in the context of single-field inflation if one calculates the variance of $q_{3}$ instead of just a single term.

\section{Infrared divergences in single-field inflation}

The computation of $\operatorname{Var}\left(\varphi \nabla_{\mathbf{x}}^{2} \varphi\right)$ is an important step in determining the observable deceleration parameter. Nevertheless, we cannot observe $\varphi \nabla_{\mathbf{x}}^{2} \varphi$ directly, rather what we observe is $q_{3}$. Therefore we want to know
$\operatorname{Var} q_{3}$. This section examines the contribution of the very long-wavelength modes to $\operatorname{Var} q_{3}$ predicted by single-field inflation. We are especially interested in the case of $n_{s} \leq 1$, where the variance $I_{-1} \equiv \operatorname{Var} \varphi$ is infrared divergent: $I_{-1} \rightarrow+\infty$.

In this section, we retain the terms from Eq. (74) that have no factors of $k_{L}$, i.e. we drop $\varphi_{L, i} \varphi_{S, i}$, and are no higher than second order in $\varphi_{L}$, which is the order at which BMR found an infrared divergence. BMR argued that the $\varphi_{L, i} \varphi_{S, i}$ term is subdominant and in any case the variance of $\varphi_{L, i}$ has no infrared divergence for $n_{s} \leq-1$. The equation for $q_{3}$ is then

$$
q_{3}=\frac{1}{2}+H_{0}^{-2}\left(\frac{5}{9}+\frac{50}{27} \varphi_{L}+\frac{250}{81} \varphi_{L}^{2}\right) \nabla_{\mathbf{x}}^{2} \varphi_{S}
$$

In order to determine the mean and variance of $q_{3}$ we need a measure. The two most obvious measures are the coordinate measure $d^{3} \mathbf{x}$ and the measure $d N$ associated with the number $N$ of CDM particles. Since at early times the CDM density is constant in the synchronous comoving gauge, it follows that $d N$ is proportional to the volume element at early times,

$$
d N \propto e^{-3 \Psi\left(x^{i}, t=0\right)} d^{3} \mathbf{x}=e^{-5 \varphi\left(x^{i}\right)} d^{3} \mathbf{x} .
$$

We can unify these two measures by working with the measure $d \mu$ defined by

$$
d \mu=\left[1+\sum_{j=1}^{\infty} \nu_{j} \varphi^{j}(\mathbf{x})\right] d^{3} \mathbf{x} \equiv J d^{3} \mathbf{x},
$$

where $\nu_{j}$ are constants. The choice $\nu_{j}=0$ corresponds to the coordinate measure, and $\nu_{j}=(-5)^{j} / j$ ! corresponds to the CDM particle number measure, up to an overall constant that does not affect the averages. The function $J$ is the Jacobian.

In order to compute the mean and variance of $q_{3}$ in Eq. (77), we must compute some averaged values involving $\varphi_{L}$ and $\nabla^{2} \varphi_{S}$. The values linear in $\varphi_{S}$ that we need are

$$
\left\langle\nabla_{\mathbf{x}}^{2} \varphi_{S}\right\rangle_{\mathbf{x}}=0,
$$

which holds by spatial homogeneity;

$$
\left\langle\varphi_{L} \nabla_{\mathbf{x}}^{2} \varphi_{S}\right\rangle_{\mathbf{x}}=0,
$$

which results from $\varphi_{L}$ and $\varphi_{S}$ having different wave numbers; and

$$
\left\langle\varphi_{L}^{2} \nabla_{\mathbf{x}}^{2} \varphi_{S}\right\rangle_{\mathbf{x}}=0,
$$

which results from the small-scale wave numbers being very large compared to the large-scale wave numbers so that the triangle inequality cannot be satisfied. Note that these averages are all taken with respect to the coordinate measure (we convert to $d \mu$ later).

We will also need the average values $\left\langle\varphi_{L}^{n}\left(\nabla_{\mathbf{x}} \varphi_{S}\right)^{2}\right\rangle_{\mathbf{X}}$ that are quadratic in $\varphi_{S}$. To write these, we follow KMNR in defining 


$$
I_{\vartheta}=\int k^{\vartheta+1} P_{\varphi}^{(0)}(k) \frac{d^{3} \mathbf{k}}{(2 \pi)^{3}},
$$

where $P_{\varphi}^{(0)}(k)$ is the power spectrum of $\varphi$ calculated by standard first-order perturbation theory in inflation (i.e., with only quadratic terms in the perturbation Lagrangian). In what follows we will consider only the long-wavelength contributions to $I_{-1}$, as these are the contributions that produce the infrared divergence reported by BMR (and in any case they dominate for $n_{s} \leq 1$ ). The first-order theory power spectrum is given explicitly by [see e.g. Eq. (2.20) of Ref. [32]]

$$
P_{\varphi}^{(0)}(k)=\frac{288 \pi^{4} G H_{*}^{4}}{25 k^{3} \dot{\phi}_{*}^{2}},
$$

where $H_{*}$ is the Hubble rate when the mode $k$ becomes super-Hubble and $\dot{\phi}_{*}$ is the rate of change of the inflaton field $\phi$ at that time. (Note that our $\varphi$ is denoted by $-\frac{3}{5} \zeta$ in Ref. [32].)

The easiest average value to compute is

$$
\left\langle\varphi_{L}^{2}\left(\nabla_{\mathbf{x}} \varphi_{S}\right)^{2}\right\rangle_{\mathbf{x}}=\left\langle\varphi_{L}^{2}\right\rangle_{\mathbf{x}}\left\langle\left(\nabla_{\mathbf{x}} \varphi_{S}\right)^{2}\right\rangle_{\mathbf{x}}=I_{-1} I_{3},
$$

which results from decoupling of the long- and shortwavelength modes. The integral $I_{3}$ is dominated by short wavelengths, and (for $\left.n_{s} \leq 1\right) I_{-1}$ by long wavelengths. The average values with 0 or 1 power of $\varphi_{L}$ are however more complicated because of corrections for the nonGaussianity of $\varphi$. The average value with 1 power of $\varphi_{L}$ and 2 of $\varphi_{S}$ is determined from the bispectrum configuration of $\varphi$ where the Fourier-space triangle has one short and two long sides, $k_{3} \ll k_{1} \approx k_{2}$. This configuration has been computed by Ref. [32] (see Eq. 4.7 in that paper) and is

$$
\left\langle\varphi_{\mathbf{k}_{1}} \varphi_{\mathbf{k}_{2}} \varphi_{\mathbf{k}_{3}}\right\rangle=\frac{5}{3}\left[n_{s}^{(0)}\left(k_{1}\right)-1\right] P_{\varphi}^{(0)}\left(k_{1}\right) P_{\varphi}^{(0)}\left(k_{3}\right) \delta\left(\sum_{j=1}^{3} \mathbf{k}_{j}\right),
$$

where $n_{s}^{(0)}\left(k_{1}\right)$ is the scalar spectral index [33]. This implies

$$
\left\langle\varphi_{L}\left(\nabla_{\mathbf{x}} \varphi_{S}\right)^{2}\right\rangle_{\mathbf{x}}=\frac{5}{3} I_{-1} \int\left[n_{s}^{(0)}\left(k_{1}\right)-1\right] k^{4} P_{\varphi}^{(0)}(k) \frac{d^{3} \mathbf{k}}{(2 \pi)^{3}} .
$$

The average $\left\langle\left(\nabla_{\mathbf{x}} \varphi_{S}\right)^{2}\right\rangle_{\mathbf{x}}$ at first glance appears to be just $I_{3}$. However we require the lowest-order correction due to long-wavelength perturbations. Thus one must return to the derivation of Eq. (86) in order to obtain the correct result. The argument provided by Ref. [32] for this situation is that the small-scale modes $\varphi_{S}$ are unaffected by large-scale modes except that these large-scale modes rescale when $k_{S}$ becomes super-Hubble. In particular, for fixed large-scale perturbation $\varphi_{L}$, the small-scale modes exit the horizon $\zeta_{L}=-\frac{5}{3} \varphi_{L} e$-folds of inflation earlier than in the unperturbed case. Therefore their power spectrum, conditioned on $\varphi_{L}$, is

$$
\begin{aligned}
\left.\frac{k^{3}}{2 \pi^{2}} P_{\varphi}(k)\right|_{\varphi_{L}} & =\frac{\left(k e^{-\zeta_{L}}\right)^{3}}{2 \pi^{2}} P_{\varphi}^{(0)}\left(k e^{-\zeta_{L}}\right) \\
& =\frac{\left(k e^{5 \varphi_{L} / 3}\right)^{3}}{2 \pi^{2}} P_{\varphi}^{(0)}\left(k e^{5 \varphi_{L} / 3}\right) .
\end{aligned}
$$

If we want the power spectrum of the small-scale modes averaged over all regions of space, then one needs to average Eq. (88) over the probability distribution of $\varphi_{L}$. Taylor-expanding Eq. (88) to second order in $\varphi_{L}$ and doing the averaging yields

$$
\begin{aligned}
\frac{k^{3}}{2 \pi^{2}} P_{\varphi}(k)= & \frac{k^{3}}{2 \pi^{2}} P_{\varphi}^{(0)}(k)+\frac{\left\langle\varphi_{L}^{2}\right\rangle_{\mathbf{x}}}{2} \frac{d^{2}}{d \varphi_{L}^{2}} \\
& \times\left[\frac{\left(k e^{5 \varphi_{L} / 3}\right)^{3}}{2 \pi^{2}} P_{\varphi}^{(0)}\left(k e^{5 \varphi_{L} / 3}\right)\right],
\end{aligned}
$$

where the second derivative term comes from the variance of $\varphi_{L}$, and there is no first derivative term because $\left\langle\varphi_{L}\right\rangle_{\mathbf{x}}=0$. The variance of $\varphi_{L}$ is $\left\langle\varphi_{L}^{2}\right\rangle_{\mathbf{x}}=I_{-1}$. If we define the variable

$$
\begin{aligned}
\tilde{\alpha}_{s}^{(0)}(k) & =\frac{1}{k^{3} P_{\varphi}^{(0)}(k)} \frac{d^{2}}{(d \ln k)^{2}}\left[k^{3} P_{\varphi}^{(0)}(k)\right] \\
& =\alpha_{s}^{(0)}(k)+\left[n_{s}^{(0)}(k)-1\right]^{2},
\end{aligned}
$$

which is related to (but different from) the scalar running $\alpha_{s}^{(0)}(k)$, Eq. (89) simplifies to

$$
\left\langle\left(\nabla^{2} \varphi_{S}\right)^{2}\right\rangle=\int k^{4} P_{\varphi}^{(0)}(k)\left[1+\frac{25}{18} \tilde{\alpha}_{s}^{(0)}(k) I_{-1}\right] \frac{d^{3} \mathbf{k}}{(2 \pi)^{3}} .
$$

Physically, Eq. (91) represents the "smearing" of the relation between $k$ and physical scale due to the variance of $\varphi_{L}$. This smears the power spectrum $P_{\varphi}(k)$. Thus it is not surprising that Eq. (91) contains a second derivative of the power spectrum. The smearing effect can be thought of as a loop correction to the scalar power spectrum in the sense that it involves an integration over an undetermined momentum (that of $\varphi_{L}$, which is packaged into $I_{-1}$ here).

The above results are most easily expressed if we define the integrals

$$
I_{\vartheta, \beta}=\int k^{\vartheta-2}\left\{\partial_{\ln k}^{\beta}\left[k^{3} P_{\varphi}^{(0)}(k)\right]\right\} \frac{d^{3} \mathbf{k}}{(2 \pi)^{3}} .
$$

In particular,

$$
I_{3,1}=\int k^{4}\left[n_{s}^{(0)}(k)-1\right] P_{\varphi}^{(0)}(k) \frac{d^{3} \mathbf{k}}{(2 \pi)^{3}}
$$

and

$$
I_{3,2}=\int k^{4} \tilde{\alpha}_{s}^{(0)}(k) P_{\varphi}^{(0)}(k) \frac{d^{3} \mathbf{k}}{(2 \pi)^{3}}
$$


Then the average values containing $\left(\nabla_{\mathbf{x}}^{2} \varphi_{S}\right)^{2}$ are

$$
\begin{aligned}
\left\langle\left(\nabla^{2} \varphi_{S}\right)^{2}\right\rangle & =I_{3}+\frac{25}{18} I_{3,2} I_{-1}, \\
\left\langle\varphi_{L}\left(\nabla^{2} \varphi_{S}\right)^{2}\right\rangle & =\frac{5}{3} I_{3,1} I_{-1}, \quad \text { and } \\
\left\langle\varphi_{L}^{2}\left(\nabla^{2} \varphi_{S}\right)^{2}\right\rangle & =I_{3} I_{-1} .
\end{aligned}
$$

Finally we come to the issue of the mean and variance of $q_{3}$. The mean of any quantity $X$ with respect to the measure $\mu$ is related to the Jacobian $J$ via

$$
\langle X\rangle_{\mu}=\frac{\langle X J\rangle_{\mathbf{x}}}{\langle J\rangle_{\mathbf{x}}} .
$$

We have evaluated $q_{3}$ including all terms of order $\varphi_{L}^{a} \varphi_{S}^{b}$, where $a \leq 2$ and $b \leq 1$. Therefore we can calculate the mean $\left\langle q_{3}\right\rangle$ only up to order $\varphi_{L}^{2} \varphi_{S}$. In principle with the Taylor series cut off in this way we can only evaluate $\operatorname{Var} q_{3}$ to this order as well. However, if one switches the variables to $Q_{3}=q_{3}-\frac{1}{2}$, then it turns out that the Taylor expansion of $Q_{3}$ in $\varphi_{L}$ and $\varphi_{S}$ contains no terms zeroth order in $\varphi_{S}$. This fact allows us to compute $\operatorname{Var} Q_{3}$ and hence $\operatorname{Var} q_{3}$ to $\operatorname{order} \varphi_{L}^{2} \varphi_{S}^{2}$.

We now calculate $\left\langle q_{3}\right\rangle_{\mu}$ to first order in $\varphi_{S}$ and $\varphi_{L}$ :

$$
\left\langle q_{3}\right\rangle_{\mu}=\frac{1}{2}+H_{0}^{-2} \frac{\left\langle J\left(\frac{5}{9}+\frac{50}{27} \varphi_{L}+\frac{250}{81} \varphi_{L}^{2}\right) \nabla_{\mathbf{x}}^{2} \varphi_{S}\right\rangle_{\mathbf{x}}}{\langle J\rangle_{\mathbf{X}}} .
$$

Now if we are dropping all terms second order in $\varphi_{S}$, then $J$ can be considered a function of $\varphi_{L}$ for the purposes of evaluating the numerator. Thus the numerator in Eq. (97) is the mean value of $\nabla_{\mathbf{x}}^{2} \varphi_{S}$ times a function of $\varphi_{L}$. Since we are working only to order $\varphi_{L}^{2}$, and we know that $\left\langle\varphi_{L}^{n} \nabla_{\mathbf{x}}^{2} \varphi_{S}\right\rangle_{\mathbf{x}}=0$, it follows that the numerator in Eq. (97) vanishes. Therefore

$$
\left\langle q_{3}\right\rangle_{\mu}=\frac{1}{2}+O\left(k_{L}, \varphi_{S}^{2}, \varphi_{L}^{3}\right) .
$$

We will compute $\operatorname{Var} q_{3}$ to first order in the power spectrum of the long-wavelength modes or to second order in the long-wavelength modes themselves, i.e. to order $\varphi_{L}^{2}$. We will also work to second order in $\varphi_{S}$. Using Eq. (98), and defining $Q_{3}=q_{3}-\frac{1}{2}$, we find

$$
\operatorname{Var} q_{3}=\left\langle Q_{3}^{2}\right\rangle_{\mu}-\left\langle Q_{3}\right\rangle_{\mu}^{2}=\left\langle Q_{3}^{2}\right\rangle_{\mu}-O\left(k_{L}^{2}, \varphi_{S}^{4}, \varphi_{L}^{6}\right) .
$$

That is, the term $\left\langle Q_{3}\right\rangle_{\mu}^{2}$ cannot contribute at order $\varphi_{S}^{2}$ even though we have only computed $\left\langle Q_{3}\right\rangle_{\mu}$ to order $\varphi_{S}$. Then

$$
\operatorname{Var} q_{3}=\frac{\left\langle J Q_{3}^{2}\right\rangle_{\mathbf{x}}}{\langle J\rangle_{\mathbf{x}}}
$$

The denominator is

$$
\begin{aligned}
\langle J\rangle_{\mathbf{x}} & =\left\langle 1+\nu_{1}\left(\varphi_{L}+\varphi_{S}\right)+\nu_{2}\left(\varphi_{L}^{2}+2 \varphi_{L} \varphi_{S}\right)\right\rangle_{\mathbf{x}} \\
& =1+\nu_{2} I_{-1} .
\end{aligned}
$$

The numerator is

$$
\begin{aligned}
\left\langle J Q_{3}^{2}\right\rangle_{\mathbf{x}}= & \frac{25 I_{3}}{81 H_{0}^{4}}+\frac{625 I_{3,2} I_{-1}}{1458 H_{0}^{4}}+\frac{2500 I_{3,1} I_{-1}}{729 H_{0}^{4}}+\frac{5000 I_{3} I_{-1}}{729 H_{0}^{4}} \\
& +\frac{125 \nu_{1} I_{3,1} I_{-1}}{243 H_{0}^{4}}+\frac{500 \nu_{1} I_{3} I_{-1}}{243 H_{0}^{4}}+\frac{25 \nu_{2} I_{3} I_{-1}}{81 H_{0}^{4}} .
\end{aligned}
$$

The physical origin of the terms in Eq. (102) is as follows: the first term is the usual variance of the deceleration due to some patches of the universe being over- or underdense. The second term is the smearing loop correction described above. The third term is a correlation between the order $\nabla_{\mathbf{x}}^{2} \varphi_{S}$ and $\varphi_{L} \nabla_{\mathbf{x}}^{2} \varphi_{S}$ in $q_{3}$ [Eq. (77)] that arises from the nonvanishing bispectrum from inflation. The fourth term comes from two places: the variance of the $\varphi_{L} \nabla_{\mathrm{x}}^{2} \varphi_{S}$ term (which is the infrared-divergent term identified by BMR) and the correlation between $\nabla_{\mathbf{x}}^{2} \varphi_{S}$ and $\varphi_{L}^{2} \nabla_{\mathbf{x}}^{2} \varphi_{S}$ terms in Eq. (77). In this case, the variance term contributes a coefficient of 2500/729 and the correlation contributes $2500 / 729$, yielding the total coefficient of 2500/729. The fifth, sixth, and seventh terms represent the modulation of the earlier terms by the noncoordinate measure.

Looking at Eqs. (101) and (102), it appears at first glance that $I_{-1}$ affects $\operatorname{Var} q_{3}$ in a highly nontrivial way depending on the values of $I_{3}, I_{3,1}$, and $I_{3,2}$. This is in fact not the case, because $I_{3}, I_{3,1}$, and $I_{3,2}$ are not independent quantities. Integration by parts in the definition [Eq. (92)] and dropping boundary terms gives

$$
I_{\vartheta, \beta}=-(\vartheta+1) I_{\vartheta, \beta-1} .
$$

Noting that the definitions imply $I_{3}=I_{3,0}$, we conclude that $I_{3,1}=-4 I_{3}$ and $I_{3,2}=16 I_{3}$. One might object that the boundary terms cannot be neglected because $I_{3}$ is ultraviolet divergent. Of course, if this divergence is not regulated, $I_{3}=\infty$, in which case even the first-order perturbation theory result for $\operatorname{Var} q_{3}$ is infinite and it makes little sense to talk about higher-order corrections. Physically, the divergence is regulated by putting in some cutoff in wave number $k$. In the case of KMNR, the regulator is placed at the horizon scale, $k \sim H_{0}$, where one separates superHubble perturbations from the sub-Hubble modes (the latter are considered to be observable perturbations to the universe, rather than a correction to the observed scale factor). In the case of deceleration parameter measurements from SNe Ia, the cutoff scale is roughly of the order of the distance $D$ to the supernovae; modes with wavelengths less than this cannot be treated by a local deceleration parameter based on the second derivative of the $d_{L}-z$ relation at $z=0$. In either case, the cutoff occurs at a fixed physical scale (the horizon scale or the distance to the supernovae), rather than at a fixed coordinate scale $\mathbf{x}$. Therefore the position of the cutoff in terms of coordinate wave number $k$ is modulated by $\varphi_{L}$ as $k_{\text {cutoff }} \propto e^{-5 \varphi_{L} / 3}$. Since this is the same as the modulation of the wave 
number in the inflationary power spectrum $P_{\varphi}(k)$ by largescale modes [see Eq. (88)], one can simply absorb the cutoff into $P_{\varphi}^{(0)}(k)$. Then $I_{3}$ becomes finite and Eq. (103) is valid.

Substituting our results that $I_{3,1}=-4 I_{3}$ and $I_{3,2}=16 I_{3}$ into Eq. (102) reduces it to

$$
\left\langle J Q_{3}^{2}\right\rangle_{\mathbf{x}}=\frac{25 I_{3}}{81 H_{0}^{4}}\left(1+\nu_{2} I_{-1}\right)
$$

and hence

$$
\operatorname{Var} q_{3}=\frac{25 I_{3}}{81 H_{0}^{4}}
$$

independently of the value of $I_{-1}$. The long-wavelength contribution to $I_{-1}$, which is responsible for the infrareddivergent terms, cancels out when all contributions are included. This is true for both the coordinate and the particle number measure. This specific example confirms the general conclusions based on causality found above.

\section{Interpretation}

The correspondence between our results and BMR reveals the origin of the infrared divergence: it comes from the assumption that $\varphi_{S}$ was taken as a function of $\mathbf{x}$ rather than $\mathbf{y}$. Since the $\mathbf{x}$ coordinate system is distorted by superhorizon perturbations, these superhorizon modes distort structures within the horizon and affect the luminosity distance. Whether this effect is observable depends on whether the values of $\varphi_{S}$ within the horizon are a Gaussian random field with the usual power spectrum $P_{\varphi}(k)$ in $\mathbf{x}$ or in $\mathbf{y}$. This can be answered only by a theory of the initial conditions. If the initial conditions are set by single-field inflation, then the fluctuations $\varphi_{L}$ that determine the relation between the coordinates $\mathbf{x}$ and $\mathbf{y}$ are set down when these large scales leave the horizon. They then become classical, and later on (i.e. many $e$ folds of inflation later) the perturbations $\varphi_{S}$ are generated. Since the generation of $\varphi_{S}$ must be causal, one would expect that within regions small compared to the wavelength of $\varphi_{L}$, inflation generates $\varphi_{S}$ homogeneous and isotropic in the $\mathbf{y}$ coordinate system. If the calculation is done in the $\mathbf{x}$ coordinate system, as we did in Sec., then the infrared divergence from the second-order perturbation theory found by BMR cancels against three other infrared divergences: one arising from the correlation of first- and third-order perturbation terms, one from the correlation of first- and secondorder perturbation terms that arises from the primordial bispectrum, and one from loop corrections to the power spectrum predicted by inflation. Physically, the disappearance of $I_{-1}$ from the statistical properties of observables such as $\operatorname{Var} q_{3}$ is a manifestation of the fact that inflation wipes out initial conditions: the later stages of inflation prevent one from observing the preexisting larger-scale structure of the universe, including the perturbations gen- erated during the early stages of inflation. (Recall that this is also how inflation solves the flatness and homogeneity problems.)

The scenario proposed by KMNR is closely related to the infrared divergence. Specifically, they argue that the $e^{10 \varphi_{L} / 3} \nabla_{\mathbf{x}}^{2} \varphi_{S}$ term in Eq. (74) has a large variance that causes the deceleration parameter to also have a large variance. At second order in perturbation theory, this term is the infrared-divergent $\varphi_{L} \nabla_{\mathbf{x}}^{2} \varphi_{S}$ found by BMR, which we find to be canceled by other divergences that were not considered by BMR. KMNR uses the full prefactor $e^{10 \varphi_{L} / 3}$ and hence includes the divergence associated with the $\varphi_{L}^{2} \nabla_{\mathbf{x}}^{2} \varphi_{S}$ term, but does not consider the divergences from the bispectrum or the loop correction to the power spectrum. From the perturbative calculation of Sec. IV C, it is not obvious whether this cancellation extends to arbitrary order. However if the later $e$-folds of inflation produce perturbations homogeneous and isotropic with power spectrum $P_{\varphi}^{(0)}(k)$ in the $\mathbf{y}$ coordinate system, as must happen on account of causality, then we need only consider Eq. (72) to realize that the superhorizon structure is irrelevant, and the cancellation of the infrared divergences that arise in the $\mathbf{x}$ coordinate system must be exact.

[As noted in Sec. II, KMNR use $q_{1}$ rather than $q_{3}$ as their deceleration parameter. As far as Eq. (74) is concerned, this does not matter since Eq. (71) is valid for $q_{1}$ as well as for $q_{3}$. To see this, note that in first-order perturbation theory, if one does a spherical expansion of the perturbation around the observer, symmetry implies that only the $l=0$ modes contribute to $q_{1}$ or $q_{3}$. If only the $l=0$ modes are present then we have $q_{3^{\prime}}$ independent of direction $n^{i}$ and $q_{3}=q_{4}$. Since $H_{1}=H_{4}$, a comparison of Eqs. (17) and (38) with the assumption that $\sigma=\omega=0$ from spherical symmetry implies $q_{1}=q_{4}$. We then have $q_{1}=q_{3}$ by transitivity. Hence Eq. (71) and thus Eq. (74) are valid for $q_{1}$ instead of $q_{3}$.]

\section{CONCLUSIONS}

In this paper, we have investigated the KMNR explanation for the accelerating expansion of the universe, which suggests that (i) in sufficiently underdense regions of the universe, the Hubble expansion appears to accelerate $\left(q_{1}<\right.$ 0 ) even with only normal matter present and Einstein gravity; and (ii) the variance $\operatorname{Var} q_{1}$ of the deceleration parameter is much greater than the simple calculation $\sim\left(10^{-5}\right)^{2}$ because of the influence of perturbation modes with wavelengths many orders of magnitude larger than the Hubble length, so that the acceleration $q_{1}<0$ has a nonnegligible probability of actually occurring. We have shown that suggestion (i) is ruled out by the Raychaudhuri equation, i.e. with GR and the SEC one always has $q_{1} \geq 0$. We have also shown that suggestion (ii) is not true for the perturbations generated by singlefield inflation, which does indeed predict $\operatorname{Var} q_{1}$ of order $I_{3}^{2} \sim\left(10^{-5}\right)^{2}$ (assuming an ultraviolet cutoff at the Hubble 
scale, as in KMNR). Therefore the KMNR mechanism cannot explain the acclerating expansion of the universe.

What freedom is there to construct models related to KMNR that do explain the acceleration of the universe with superhorizon perturbations? Of the two elements of the KMNR mechanism, (ii) may be the easiest to circumvent. In single-field inflation, the perturbations laid down by the early stages of inflation are adiabatic so the perturbations laid down by the later stages of inflation must "look the same" (i.e. have the same power spectrum in our $\mathbf{y}$ coordinate system) everywhere. This requirement is the physics underlying the particular limiting forms for the bispectrum and the one-loop correction to the power spectrum that we used in Sec. IV C to argue that single-field inflation produces no infrared divergences. In contrast, multifield inflation models can produce isocurvature modes so that different patches of the universe look different. In this case $\operatorname{Var} q_{1}$ may be large because one takes the variance over regions with a different mix of cosmological fluids [25]. Even then, however, an observer can only see one Hubble volume because of causality and so $\operatorname{Var} q_{1}$ is not an observable, rather it is the variance of a distribution from which one obtains a single sample. In such a case the only role played by the superhorizon perturbations is to alter the initial conditions: all observables, including $q_{1}$, are obtained from causal evolution that can be calculated from knowledge of the perturbations within the observer's horizon. More importantly for the dark energy question, the combination of GR and the SEC still forbids $q_{1}<0$.

If one is to find a way to keep GR, the SEC, zero vorticity, and neglect perturbations with wavelengths small compared to the Hubble length, and still maintain consistency with the observational results, one is forced to find a situation in which $q_{1}$ as defined above is not what is actually measured in the SN Ia experiments. BMR argued that $q_{3}$ is a better representation of what is observed, since it is based on luminosity distances; the concordance cosmology has $q_{3} \sim-0.6$. Unfortunately, we found in Sec. II that $q_{3}<0$ is possible within the GR+SEC framework only for anisotropic expansion, since the angular average $\left\langle H_{3^{\prime}}^{2} q_{3^{\prime}}\right\rangle_{4 \pi}$ must be non-negative. In this case, there must also be lines of sight along which $q_{3^{\prime}}>0$. There is no observational evidence that this is the case. Indeed, it would be an extraordinary mystery to have the deceleration parameter $q_{3^{\prime}}$ vary by an amount of order unity on scales comparable to the Hubble length and still produce a CMB isotropic to the level of a few parts in $10^{5}$.

We conclude that cosmological models based on GR with irrotational initial conditions and perturbations only at and above the Hubble scale and only matter fields that conform to the SEC cannot explain the accelerating expansion. This paper does not exclude the possibility of using backreaction from sub-Hubble perturbations to explain the accelerating expansion. The latter possibility is difficult to investigate as it involves complicated nonlinear physics; the perturbative calculations [34], which account for the nonlinear evolution of density perturbations, but not for strong field GR effects, suggest that the sub-Hubble backreaction is small [35]. Nevertheless, only a full nonperturbative analysis would be definitive since there are rare regions of the universe such as black holes that cannot be described as a perturbation of an FRW spacetime. Regardless of the sub-Hubble physics, however, the superhorizon perturbations are not a viable mechanism to explain the acceleration of the universe: evolution of perturbations that lie beyond the horizon in real space cannot affect observables, and the superhorizon perturbations can only act via their effects on the initial conditions within our horizon. These effects are then constrained by the "no go" theorems that require $q_{1}$ and $q_{4}$ to be nonnegative unless one invokes vorticity, modified gravity, or dark energy.

\section{ACKNOWLEDGMENTS}

C. H. wishes to thank Latham Boyle and Mustapha Ishak for enlightening discussions. C. H. is supported by NASA NGT5-50383. U.S. is supported by Packard Foundation, NASA NAG5-11489 and NSF CAREER-0132953.

\section{APPENDIX A: CONVERGENCE RADIUS OF SERIES FOR $\Psi$}

The purpose of this appendix is to investigate the convergence properties of the power series Eq. (52) for $\Psi$ in the model of Sec. III. Our framework will be the result from complex analysis that a Taylor series of an analytic function has a radius of convergence given by the distance to the nearest singularity. We begin by defining the dimensionless scale factor

$$
\beta=\frac{C a}{H_{0}^{2}}=\left(\frac{3 t}{4 \Xi}\right)^{2 / 3},
$$

so that Eq. (45) becomes

$$
a_{\mathrm{open}}=\Xi C^{1 / 2}(\cosh \eta-1)
$$

and

$$
\beta^{3 / 2}=\frac{3}{4}(\sinh \eta-\eta)
$$

It will be most convenient for the purposes of this appendix to treat Eq. (52) as a Taylor series in the dimensionless variable $\beta$ rather than $a$ or $C$. If we determine the radius of convergence in $\beta$, then this immediately yields the radius of convergence in $a$ or $C$.

We want to determine the singularities of the function $\Psi$ of Eq. (49) or equivalently $\ln \left(a_{\text {open }} / a\right)$. First we comment on the analytical structure near $\beta=0$. The function $\eta(\beta)$ defined by Eq. (A3) is double-valued near zero, with two solutions $\eta_{1}$ and $-\eta_{1}$. This presents no problem because both result in a single value for $a_{\text {open }}$. Specifically, near 
$\beta=\eta=0$, we have $\frac{3}{4}(\sinh \eta-\eta) \sim \frac{1}{8} \eta^{3}$ so $\eta \sim 2 \beta^{1 / 2}$ and

$$
a_{\text {open }} \sim \Xi C^{1 / 2} \frac{\eta^{2}}{2} \sim 2 \Xi C^{1 / 2} \beta \sim \frac{H_{0}^{2}}{C} \beta .
$$

Thus $a_{\text {open }}$ is well behaved at the origin (even for complex values).

Equation (52) is the Taylor expansion of Eq. (49) in $\beta$ and hence the value of $\beta$ with smallest absolute value that makes $\ln \left(a_{\text {open }} / a\right)$ singular determines the radius of convergence of Eq. (52). We can find these singularities by taking the derivative,

$$
\begin{aligned}
\frac{d}{d \beta} \ln \frac{a_{\text {open }}(\beta)}{a(\beta)} & =\frac{d}{d \beta} \ln \frac{\cosh \eta-1}{\beta} \\
& =\frac{\sinh \eta}{\cosh \eta-1} \frac{d \eta}{d \beta}-\beta^{-1} .
\end{aligned}
$$

Using the implicit derivative of Eq. (A3),

$$
\frac{3}{2} \beta^{1 / 2}=\frac{3}{4}(\cosh \eta-1) \frac{d \eta}{d \beta}
$$

we find

$$
\frac{d}{d \beta} \ln \frac{a_{\mathrm{open}}}{a}=\frac{2 \sinh \eta}{(\cosh \eta-1)^{2}} \beta^{1 / 2}-\beta^{-1} .
$$

This function appears to have singularities where $\cosh \eta=$ 1 or $\beta=0$. The singularity at $\beta=0$ is only apparent since at small values of $\beta$, we know that $\ln \left(a_{\text {open }} / a\right) \rightarrow 0$. Thus the only singularities can appear when $\cosh \eta=1$ and $\beta \neq$ 0 . The solutions to $\cosh \eta=1$ are $\eta=-2 \pi i m$ where $m$ is any integer. Any point with $\eta=-2 \pi i m, m \neq 0$ must actually be a singularity since for $\eta=-2 \pi i m+\epsilon$, we have (by periodicity of the hyperbolic functions with pe$\operatorname{riod} 2 \pi i)$

$$
\frac{2 \sinh \eta}{(\cosh \eta-1)^{2}}=\frac{2 \sinh \epsilon}{(\cosh \epsilon-1)^{2}} \sim \frac{2 \epsilon}{\left(\epsilon^{2} / 2\right)^{2}} \sim 8 \epsilon^{-3}
$$

then since $\beta^{1 / 2}$ and $\beta^{-1}$ are analytic for $\beta \neq 0$ there must be a singularity in Eq. (A7). The points with $\eta=-2 \pi i m$, $m \neq 0$ correspond to

$$
\beta^{3 / 2}=\frac{3}{4}[\sinh (-2 \pi i m)+2 \pi i m]=\frac{3 \pi i m}{2} .
$$

The values $m=0$ correspond to $\beta=0$ where there is no singularity. The other singularities occur at the points

$$
\beta=\left(\frac{3}{2} \pi\right)^{2 / 3} m^{2 / 3} E,
$$

$m \neq 0$, where $E$ is any cube root of -1 . The closest such singularities to the origin have $m= \pm 1$ and hence $|\beta|=$ $\left(\frac{3}{2} \pi\right)^{2 / 3}$. Thus we conclude that the radius of convergence of Eq. (52) is $|\beta|<\left(\frac{3}{2} \pi\right)^{2 / 3}$ or

$$
\left|C H_{0}^{-2} a\right|<\left(\frac{3}{2} \pi\right)^{2 / 3} \text {. }
$$

[1] J. L. Tonry et al., Astrophys. J. 594, 1 (2003).

[2] R. A. Knop et al., Astrophys. J. 598, 102 (2003).

[3] A. G. Riess et al., Astrophys. J. 607, 665 (2004).

[4] A. G. Riess et al., Astron. J. 116, 1009 (1998).

[5] B. J. Barris et al., Astrophys. J. 602, 571 (2004).

[6] S. Perlmutter et al., Astrophys. J. 517, 565 (1999).

[7] C.L. Bennett et al., Astrophys. J. Suppl. Ser. 148, 1 (2003).

[8] L. Verde et al., Mon. Not. R. Astron. Soc. 335, 432 (2002).

[9] M. Tegmark et al., Astrophys. J. 606, 702 (2004).

[10] M. Tegmark et al., Phys. Rev. D 69, 103501 (2004).

[11] D. J. Eisenstein et al., astro-ph/0501171.

[12] P. Fosalba, E. Gaztañaga, and F. J. Castander, Astrophys. J. Lett. 597, L89 (2003).

[13] R. Scranton et al., astro-ph/0307335.

[14] N. Afshordi, Y. Loh, and M. A. Strauss, Phys. Rev. D 69, 083524 (2004).

[15] S. Boughn and R. Crittenden, Nature (London) 427, 45 (2004).

[16] P. Fosalba and E. Gaztañaga, Mon. Not. R. Astron. Soc. 350, L37 (2004).

[17] M. R. Nolta et al., Astrophys. J. 608, 10 (2004).
[18] S. Boughn and R. Crittenden, Astrophys. J. 612, 647 (2004).

[19] N. Padmanabhan, C. M. Hirata, U. Seljak, D. Schlegel, J. Brinkmann, and D. P. Schneider, Phys. Rev. D 72, 043525 (2005).

[20] E. Barausse, S. Matarrese, and A. Riotto, Phys. Rev. D 71, 063537 (2005).

[21] E. W. Kolb, S. Matarrese, A. Notari, and A. Riotto, hep-th/ 0503117 .

[22] A. H. Guth, Phys. Rev. D 23, 347 (1981).

[23] A. Linde, Phys. Lett. B 108, 389 (1982).

[24] A. Albrecht and P. J. Steinhardt, Phys. Rev. Lett. 48, 1220 (1982).

[25] E. W. Kolb, S. Matarrese, A. Notari, and A. Riotto, astro$\mathrm{ph} / 0410541$.

[26] R. M. Wald, General Relativity (University of Chicago Press, Chicago, 1984).

[27] M. Sasaki, Mon. Not. R. Astron. Soc. 228, 653 (1987).

[28] C. W. Misner, K. S. Thorne, and J. A. Wheeler, Gravitation (W. H. Freeman and Co., San Francisco, 1973).

[29] R. K. Sheth and R. van de Weygaert, Mon. Not. R. Astron. Soc. 350, 517 (2004).

[30] J. E. Gunn and J. R. I. Gott, Astrophys. J. 176, 1 (1972). 
[31] S.W. Hawking and G.F.R. Ellis, The Large Scale Structure of Space-Time (Cambridge University Press, Cambridge, U.K., 1975).

[32] J. Maldacena, J. High Energy Phys. 5 (2003) 13.

[33] Note that " $n_{s}$ " in Ref. [32] denotes what most cosmologists call $n_{s}-1$.

[34] U. Seljak and L. Hui, in Astronomical Society of the Pacific Conference Series 88: Clusters, Lensing, and the
Future of the Universe (Astronomical Society of the Pacific, San Francisco, CA, 1996), p. 267.

[35] The possibly ultraviolet-divergent term in Ref. [36] was found by Ref. [37] to not significantly affect the expansion rate, at least at second order.

[36] S. Räsänen, J. Cosmol. Astropart. Phys. 02 (2004) 003.

[37] E. W. Kolb, S. Matarrese, A. Notari, and A. Riotto, Phys. Rev. D 71, 023524 (2005). 